\title{
Analysis the digital images by using morphology operators
}

\author{
Hadeel Amjed Saeed, Sumaya Hamad, Azmi Tawfik Hussain \\ Department of Computer Science, College of Computer Science and Information Technology, University of Anbar, \\ Anbar, Iraq
}

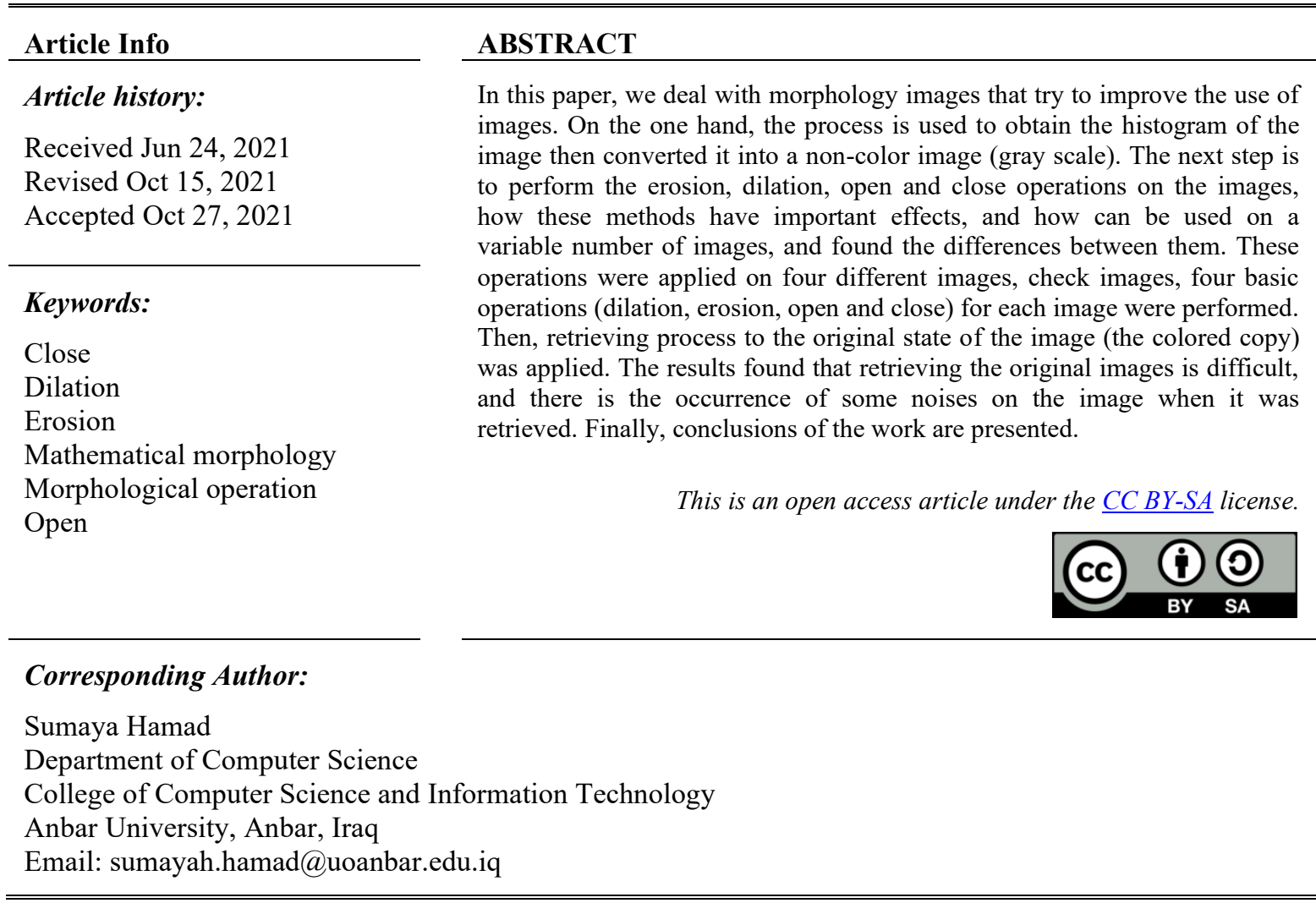

\section{INTRODUCTION}

The problem of enhancement digital images could be addressed using several approaches, one of which is mathematical morphology (MM). Such operators pick a new gray level amidset two patterns (primary) for each point of the analyzed image based on some proximity criterion [1], [2]. MM was initially designed to analyze structures geometrically. However, its strong theoretical foundation has permitted its use in a variety of disciplines, including graphs, and digital image processing to mention a few [2]-[4].

Despite the domain is old completely, using morphological operators has a regenerate benefit for different image processing tasks in new year's. This is mostly owing to their ability to solve a variety of difficult shape-related challenge [2]. Segmentation, as well as automated counting and inspection, are key areas of application. Morphology is a strong and essential collection of methods that can be mathematically handled precisely within the context of set theory. Although the advantages of mathematical rigour are provided by this set-theoretic structure, it is not easily accessible to those who are not mathematically educated, and the key ideas and applications of morphology can be grasped far more easily through a realistic and intuitive discussion [4]. Morphological operations can be applied to any image, but the most common use of morphology is for processing binary images, and the most common morphological operators are dilation and erosion. It is possible to demonstrate that many more complex morphological procedures can be reduced to dilations and erosions [1], [2], [5].

For a range of image processing applications like segmentation, filtering, and edge detection, mathematical morphology offers strong nonlinear operators. It is presented and demonstrated in several applications how to apply these non-linear operators in an end-to-end deep learning framework. In different 
cases, new layers based on morphological non-linearities that are complimentary to convolution layers are presented. These additional layers can be utilized to bring non-linear operations and pooling together in a single operation [6]. A method based on morphological signals and image processing techniques is presented to efficiently reduce noise and detect impulses. The proposed approach consists primarily of two components: a new double cross-correlation procedure for the reduction of noise and an enhanced image processing technique for identifying fault signals [7].

On the composite images of the total attenuated backscatter coefficient, an atmospheric boundary layer height $(\mathrm{ABLH})$ estimator based on image processing techniques is proposed and used. To correctly set up and modify the images to identify edges, a pre-processing step basis of morphological filters is done to the composite total backscatter image. Finally, both mathematical morphology and object-based analytics are used to post-process the detected edges [8]. A new approach for automated analysis of fluorescence microscopy images is also given, which benefits from integrating several segmentation procedures to achieve the best final segmentation [9]. It is proposed to use label thickening and thinning to enhance road extraction recall from satellite photos convolutional neural network (CNN)-based. More aggressively extract roads is prompted to the $\mathrm{CNN}$ as a result of the thicker road labels, retaining the topological roads information. The anticipated segment maps must be narrowed back to their original width after inference [10].

Also, there is a study looking into the morphological features of mature follicles with clinical significance. In 72 natural ovulation cycles, the five morphological indices of follicles have been assessed. The follicle pictures were taken the day before ovulation [11]. The model used the application of the morphological operators to design a road extraction algorithm for processing National Aeronautics and Space Administration satellite pictures, and then the detection of the roadway network. The fast stage is to increase information extraction by utilizing a variety of picture improvement methods. The next step in the design process is to extract the road characteristics from the national aeronautics and space administration (NASA) picture using an unsupervised classification approach. Then the system applies to improve morphological operator extraction [12]. A novel technique for modeling the volume-controlled invasion of a non-wetting phase into voxel pictures is proposed. The approach is used in conjunction with the classic morphological image opening approach, which simulates invasion by pressure. The method is tested upon morphological image opening and achieves near-perfect agreement at equal saturations, which is what theory predicts. The envelope of peak pressures also provides the pressure-based capillary pressure acquired by morphological opening. Therefore, the suggested algorithm's results are a superset of the morphological approach's findings [13]. In the multi-scale morphological gradient (MSMG) domain, an efficient IR and VIS picture fusion using nonsubsampled shearlet transform (NSST) and pulse-coupled neural network (PCNN) is presented. Firstly, the source images are decomposed through NSST to obtain high-frequency sub-images and low-frequency subimages. Secondly, to integrate both frequency sub-images, a PCNN with parameters controlled by the multiscale morphological gradient (MSMG) fusion technique is utilized. Lastly, an inverse NSST is utilized to recreate the merged picture [14].

An oxide transistor and a memristor-based crossbar array are used to show an effective defect detection technique with minimum power usage that may be used to neuromorphic computing. When compared to a traditional complementary metal-oxide-semiconductor (CMOS)-based digital implementation, an increased defect detection performance is effectively demonstrated using a codesigned hardware and software neuromorphic system paired with a dynamic Gaussian blur kernel operation [15]. The automated study for using image processing of root structure is required for research on rice (Oryza sativa) roots. It's difficult for a digital filter to distinguish major roots from lateral roots in the face of an opaque and crowded background. Alejandro F. Frangi introduced the initial Frangi filter (FF) in 1998. It is a low-pass filter specialized to blood vessel image improvement. The FF is used to detect the roots due to the similarities between vessels and roots. The original FF, on the other hand, simply increases the tube-like core roots while erasing the lateral roots. As a result, a novel approach is created to satisfy the demands by preserving the lateral and primary morphological structure of roots at the same time [16].

A new approach to classify hyperspectral image (HIS) with few training samples using a convolutional neural network in that context is proposed. The proposed approach employed an extended morphological profile cube (EMPC) to extract rich spectral-spatial features and then used a 3D densely connected network for classification. Besides, we used sparse principal component analysis to reduce the high spectral dimension of intensity-hue-saturation (IHS) [17].

In this work, a brief background on morphological transformation is presented on images. Approximation of the image background to the background using block analysis and adjustments to improve photographs with poor illumination. The concept of a multi-background is presented through the reconstruction of the opening. A comparison of many strategies for improving image contrast. We aim to find out the image background and boost the contrast in the poor lighting of gray level image. The first operator applies histogram and then the operator uses dilation and erosion then opening and close by reconstruction. 
Finally, the organization of this paper is as follows: section 1 describes some of the related works, section 2 contains the background of the proposed system and its concepts. Subsection 2.3 illustrates the methodology and results. Conclusions and future works are presented in section 3.

\section{BACKGROUND OF THE PROPOSED SYSTEM}

The difference between the image's highest and lowest intensity levels, or its spectrum of intensity, and it a measure of its contrast called the range of intensity. There are standard techniques to improve the degraded image poor contrast, such as histogram equalization [1], [18]. However, because image attributes differ greatly from one region to the next within a single image, and the local histogram does not always match the global histogram, context-sensitive approaches based on local contrast variance are required. The enhancement level is insignificant, and it produces good results only for certain images. It does not produce good results for all images. For the majority of the photos, especially those taken in low light [18].

\subsection{Morphology}

Morphology is an image processing technique that is focused on the shape and form of objects [2], [19]. Morphological methods use a structured element to construct an output image that is the same size as the input image. A contrast of the corresponding pixel in the input image with its neighbors determines the value of each pixel in the input image. A morphological operation may create to respond to particular shapes in the input image by adjusting the shape and size of the neighbor. The morphological operations can be described first on gray scale images with a planar source image (single-channel). After that, the concept can be extended to include full-color photographs [1].

\subsection{Morphological operations}

Morphological image analysis is frequently performed using a mixture of morphological procedures such as (erosion, dilation, opening, and closing) [4], [20]. Mathematical morphology can be defined using these operations [21]. Morphological operations apply structural features to an input image and produce a similar-sized output image. Regardless of the element's size. The origin is in the heart of the center [5].

\subsubsection{Dilation}

Dilation is a transition that results in a picture that has the same shape as the original but various size. The initial figure is extended or shrunk by dilation [1], [22]. The dilation of A by the structuring element $\mathrm{B}$ is defined by [23]:

$$
A \oplus B=U_{\mathrm{b} \in B} A
$$

When B have a center on the origin, as before; then the dilation of A by B as the center of B moves within A can be thought of as the location of the B-protected locations.

As previously mentioned, if B's center is on the root, then A's dilation by B can be interpreted as the locus of the B-covered points as B's center moves within A. $f \oplus s$ is the dilation of image $f$ caused by structuring element $\mathrm{s}$. $\mathrm{s}$ is a structuring function. This rule is used to calculate the new pixel value: located with its radix at $(\mathrm{x}, \mathrm{y})$ and by applying the rule, the new pixel is calculated [1]:

$$
g=\left\{\begin{array}{c}
1 \text { if } s \text { hits } f \\
0 \text { if otherwise }
\end{array}\right\}
$$

Take note of how structural elements define the neighborhood of the circled pixel of interest. The function of dilation applies the relevant rule on pixels in the vicinity, then the correspondent pixel value assigns in the output picture.

\subsubsection{Erosion}

Erosion used in the image to reduce the size of objects, and it is understood the erosion reduces peaks and widens minimum regions, so it can eliminate positive noise but has little effect on negative impulsive noise. The structuring factor B's erosion for the binary image A is described as follows [23]-[25]:

$$
A ! B=\left\{z \in E \mid B_{\approx} \subseteq A !\right\}
$$

If all of the pixels in a binary image are assigned to 0 , the output pixel is also assigned to $0 . \mathrm{f} \theta \mathrm{s}$ is the erosion of image $\mathrm{f}$ caused by structured element $\mathrm{s}$. The structuring factor $\mathrm{s}$ is centered at $(\mathrm{x}, \mathrm{y})$ then the value of the new pixel is calculated by applying following rule [1]: 


$$
g=\left\{\begin{array}{c}
1 \text { if } s \text { hits } f \\
0 \text { if otherwise }
\end{array}\right\}
$$

The equation above, fit implies that all pixels in the structuring factor cover on pixel in the image. The dilation function assigns a correspondent pixel value in the output image after applying the relevant rule to the pixels in the neighborhood [26].

\subsubsection{Opening} $\mathrm{B}[27]-[30]$

The opening of $\mathrm{A}$ by $\mathrm{B}$ is obtained by eroding $\mathrm{A}$ by $\mathrm{B}$ and then dilating the resulting image by

$$
A \text { о } B=(A ! B) \oplus B
$$

The opening is a square of side ten with rounded corners and a corner radius of two within the instance of a square of side ten and a disc of radius two as the structural element. The sharp edges begin to fade. Erosion which is then followed by dilation using the same structural element causes the opening of the image [26].

\subsubsection{Closing operation}

The operation of closing an image is the inverse of the operation of opening it. Lastly, The dilation of $\mathrm{A}$ by $\mathrm{B}$, accompanied by the erosion of the resulting structure by $\mathrm{B}$, results in the closure of $\mathrm{A}$ by B [25], [27], [28], [30]:

$$
\mathrm{A} \cdot \mathrm{B}=(\mathrm{A} \oplus \mathrm{B}) \mathrm{B} !
$$

\subsection{Methodology}

The first step read the image and enhancement by histogram.it is begin by Convert the RGB image into HSV image format, as show in the equation.

$$
H S V=\operatorname{rgb} 2 h s v(I)
$$

Where: I=Read image. Then perform histogram equalization on intensity component and convert to RGB.

$$
\begin{aligned}
& H e q=\operatorname{histeq}(\operatorname{HSV}(:,:, 3)) \\
& R G B=\operatorname{hsv} 2 r g b\left(H S V \_ \text {mod }\right)
\end{aligned}
$$

Then moving mask $(3 * 3)$ matrices on to the block analysis phase, in which the image is divided into several blocks, enhancement of each block being individually as shown in the Figure 1, and then the erosion and dilation method applied, which is similar to analysis of block but uses morphological operations (dilation, erosion, close and opening) rather than splitting the entire image into blocks.

These operations in morphology (erosion, dilation, close, open) are used to utilized grayscale images, which were then converted to color images by improving and concatenating them one by one individually to create the enhanced images depicted in the Algorithm 1 and Figure 2. In figures show the steps of operations and steps of proposed system to process image:
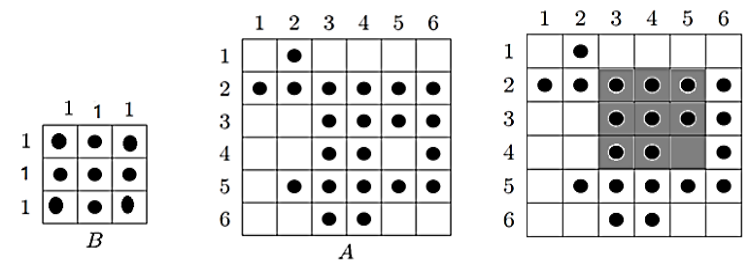

Figure 1. The block of mask and image

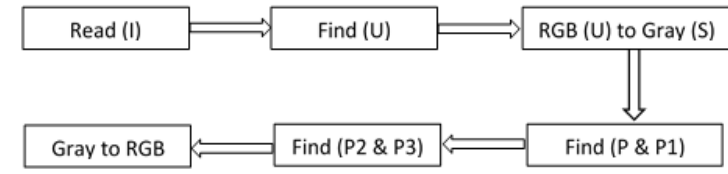

Figure 2. The block diagram of the proposed system

Algorithm 1

Input: Original image (I)

- Read image (I)

- Find the Histogram og image (I,U)

- Convert the colored image (RGB) to gray (U,S)

- Apply dilatation $\mathrm{P}=\mathrm{MAX}(\mathrm{W} \oplus \mathrm{S})$ 
- Apply erosion P1=MIN (WOS)

- Apply open P2=MIN (W•P)

- Apply close P3=MIN (WoP1)

- Convert the gray image to RGB image

\subsection{Results}

The test material used in this paper belongs basically to four different images. Four morphological operations are applied for each image as shown in the following figures. The picture can be seen in Figure 3 that when applying the histogram, the colors of the picture changed and became clearer and when applying the morphological stages, we will notice the changes that occurred to the pictures in terms of increasing the blackness or whiteness of the picture as shown in the pictures.

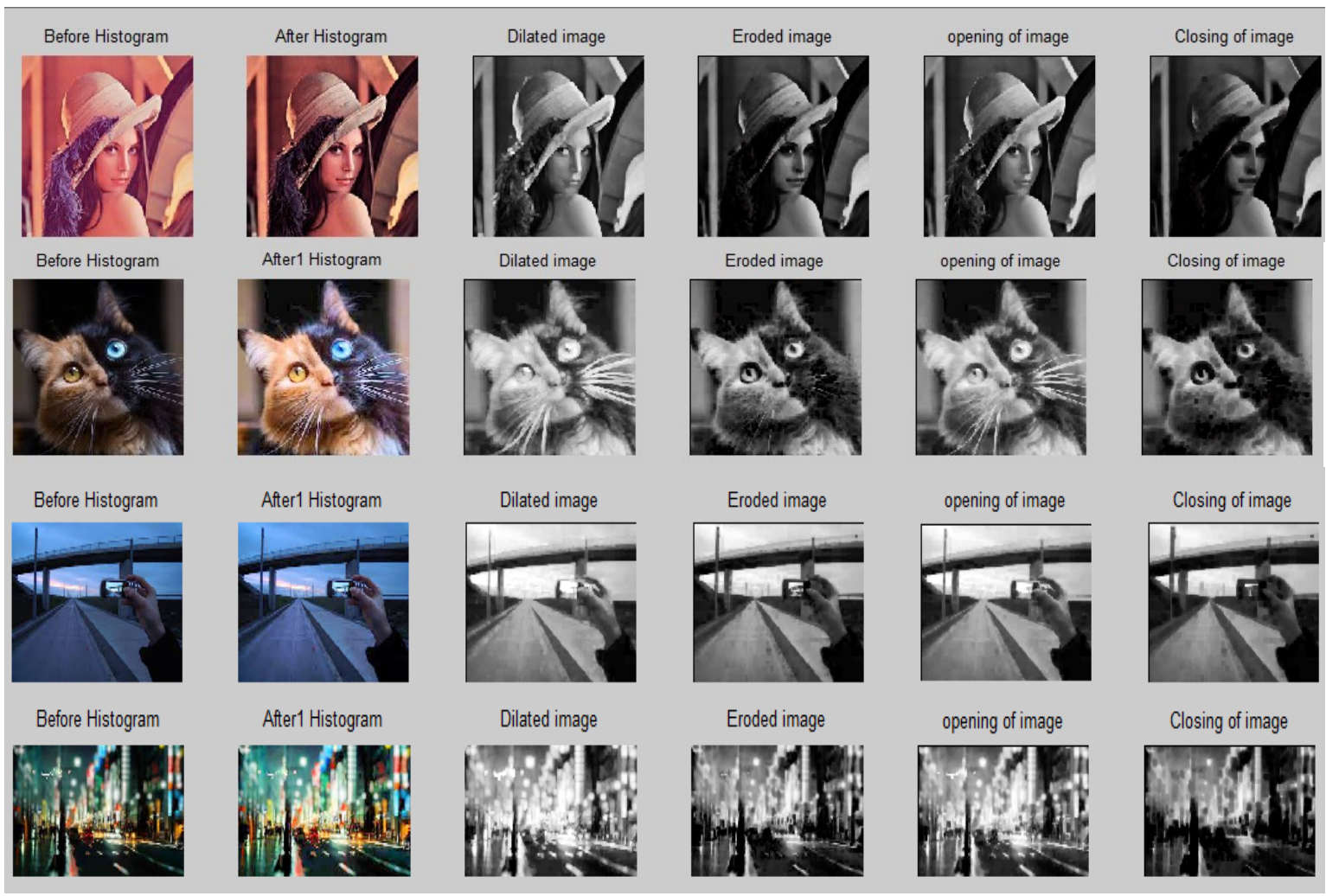

Figure 3. Four morphological images (before histogram, after histogram, dilation, erosion, open, and close)

We note that the process of retrieving the image from gray scale to the previous colors was a small percentage of coloring, and light colors were recovered more than dark colors as shown in Figure 4 (seen in Appendix). The retrieve color image its difficult and we note in the image with light color like pink with a gradation and blue with its gradation are the most visible in the retrieved photos, as in the pictures of (the bridge and Lina).

\section{CONCLUSION}

As we see, the background on morphological transformation is presented briefly. The erosion, dilation, open and close operations are performed on various and different images. The process dealing with non-color images in terms of applying morphology processes is easier than color images. But the process of converting the image from non-colored to colored image is difficult. Artificial intelligence algorithms can be used to find the best value for the colored image to improve the image's condition as future work. 


\section{APPENDIX}
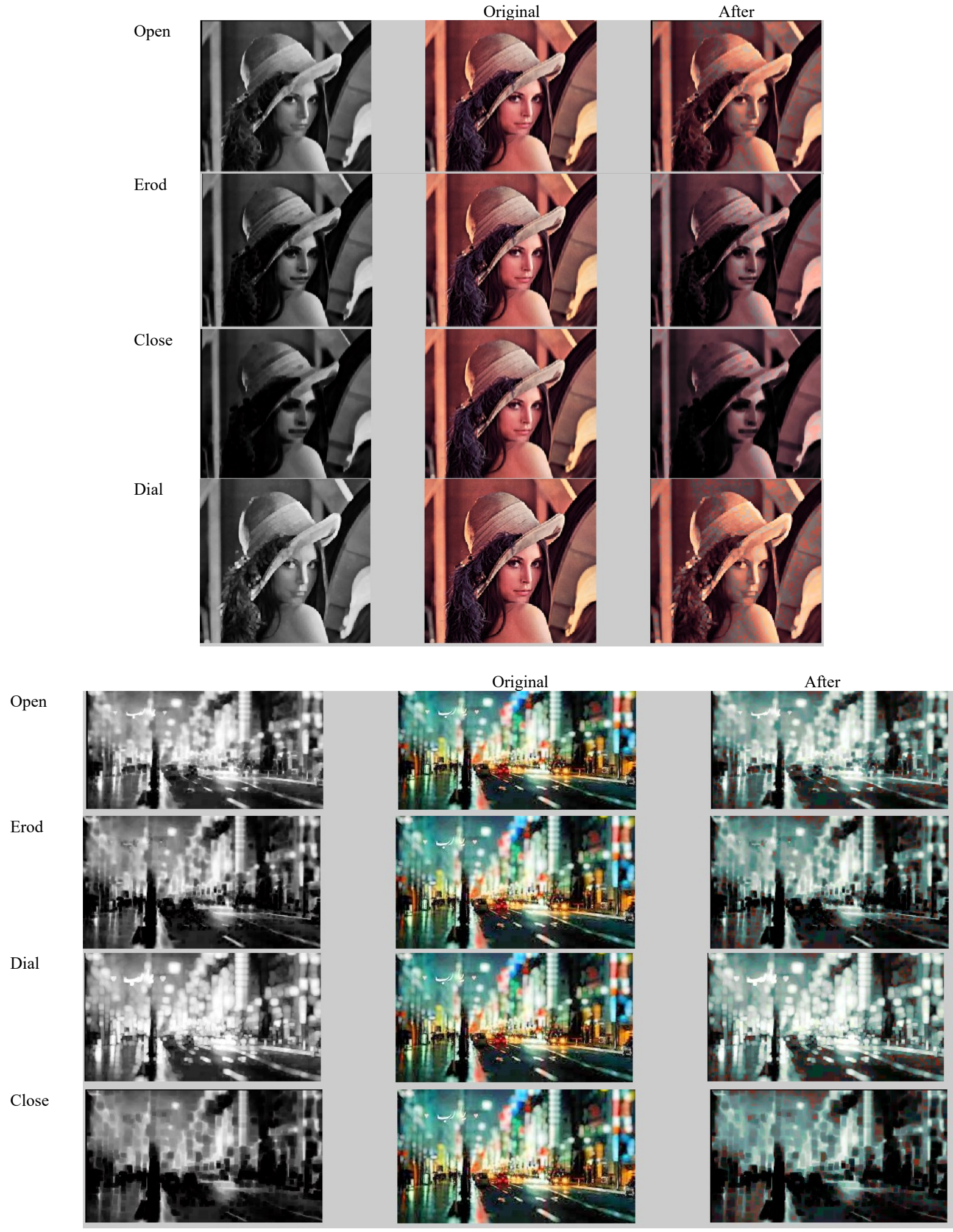

Figure 4. Four morphological images (dilation, erosion, open, and close) for four original images and retrieved colored images after operations 


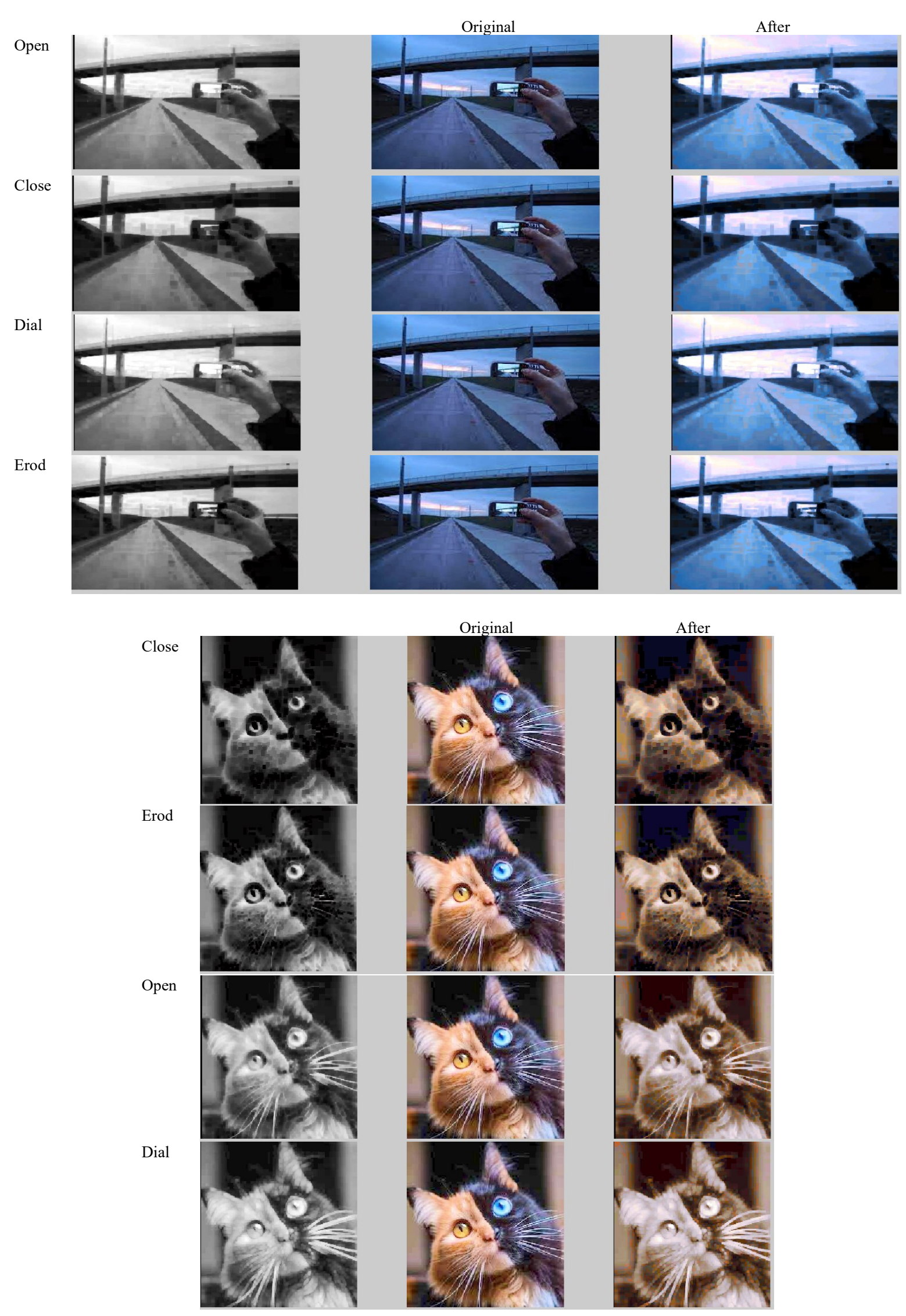

Figure 4. Four morphological images (dilation, erosion, open, and close) for four original images and retrieved colored images after operations (continue) 


\section{REFERENCES}

[1] K. Sreedhar and B. Panlal, "Enhancement of images using morphological transformation," International Journal of Computer Science \& Information Technology, vol. 4, no. 1, pp. 33-50, 2012, doi:10.5121/ijcsit.2012.4103.

[2] N. Efford, "Digital image processing a practical introduction using java (with CD-ROM)," Boston, USA: AddisonWesley Longman Publishing Co., Inc., 2000.

[3] R. Mondal, M. S. Dey, and B. Chanda, "Image Restoration by Learning Morphological Opening-Closing Network," Math. Morph.-The. Apps., vol. 4, no. 1, pp. 87-107, 2020, doi: 10.1515/mathm-2020-0103.

[4] I. R. Terol-Villalobos, "Morphological image enhancement and segmentation," Advances in Imaging and Electron Physics, vol. 118, pp. 208-271, 2001, doi: 10.1016/S1076-5670(01)80106-3.

[5] J. Serra, "Mathematical Morphology Vol. I. London," UK: Academic, 1982.

[6] G. Franchi, A. Fehri, and A. Yao, "Deep morphological networks," Pattern Recognition, vol. 102, 2020, doi: 10.1016/j.patcog.2020.107246.

[7] Y. Li, X. Liang, Y. Chen, Z. Chen, and J. Lin, "Wheelset bearing fault detection using morphological signal and image analysis," Struct. Control Heal. Monit., vol. 27, no. 11, 2020, doi: 10.1002/stc.2619.

[8] G. Vivone et al., "Atmospheric boundary layer height estimation from aerosol lidar: a new approach based on morphological image processing techniques," Atmos. Chem. Phys., vol. 21, no. 6, pp. 4249-4265, 2021, doi: 10.5194/acp-21-4249-2021.

[9] S. P. Voigt, K. Ravikumar, B. Basu, and S. R. Kalidindi, "Automated Image Processing Workflow for Morphological Analysis of Fluorescence Microscopy Cell Images," JOM, vol. 73, pp. 1-10, 2021, doi: 10.1007/s11837-021-04707-w.

[10] H. Im and H. Yang, "Improvement of CNN-Based Road Extraction from Satellite Images via Morphological Image Processing," IGARSS 2020 - 2020 IEEE International Geoscience and Remote Sensing Symposium, 2020, pp. 2559-2562, doi: 10.1109/IGARSS39084.2020.9324630.

[11] L.-F. Bao and H.-M. Li, "Measurement of the five morphological indexes of follicles using image processing toolbox," Multimed. Tools Appl., vol. 80, no. 8, pp. 12731-12735, 2021, doi: 10.1007/s11042-020-10402-w.

[12] E. P. S. Win, "Urban Road Change Detection using Morphological Processing," Qubahan Acad. J., vol. 1, no. 1, pp. 57-61, 2021, doi: 10.48161/qaj.v1n1a29.

[13] J. T. Gostick, J. Yang, and E. S. Boek, "Simulating volume-controlled invasion of a non-wetting fluid in volumetric images using basic image processing tools," arXiv Prepr. arXiv2101.03240, 2021.

[14] W. Tan, J. Zhang, P. Xiang, H. Zhou, and W. Thitøn, "Infrared and visible image fusion via nsst and penn in multiscale morphological gradient domain," in Optics, photonics and digital technologies for imaging applications $V I, 2020$, doi: 10.1117/12.2551830.

[15] H. S. Lee et al., "Efficient Defect Identification via Oxide Memristive Crossbar Array Based Morphological Image Processing," Adv. Intell. Syst., vol. 3, no. 2, 2021, doi: 10.1002/aisy.202000202.

[16] L. Gong, X. Du, C. Lin, K. Zhu, C. Liu, and W. Liang, "Automated high-resolution structure analysis of plant root with a morphological image filtering algorithm," Mathematical Problems in Engineering, 2021, doi: 10.1155/2021/4021426.

[17] A. Diakite, G. Jiangsheng, and F. Xiaping, "Extended Morphological Profile Cube for Hyperspectral Image Classification," TechRxiv, Preprint, 2021, doi: 10.36227/techrxiv.14560848.v1.

[18] O.-J. Skrede, "Morphology on binary images," University of Oslo, 2017. [Online]. Available: https://www.uio.no/studier/emner/matnat/ifi/INF2310/v17/undervisningsmateriale/slides_inf2310_s17_week15.pdf

[19] A. Y. Aidoo, M. Wilson, and G. A. Botchway, "Chest radiograph image enhancement with wavelet decomposition and morphological operations," TELKOMNIKA (Telecommunication, Computing, Electronics and Control), vol. 17 , no. 5, pp. 2587-2594, 2019, doi: 10.12928/telkomnika.v17i5.11964.

[20] R. C. Gonzalez and R. E. Woods, "Digital image processing," New Jersey, USA: Prentice Hall, 2002.

[21] S. Ramdhan and M. Syafrullah, "Fish Eggs Calculation Models Using Morphological Operation," 2019 6th International Conference on Electrical Engineering, Computer Science and Informatics (EECSI), 2019, pp. 392-397, doi: 10.23919/EECSI48112.2019.8976960.

[22] D. Campbell, "Application for morphological image processing," Computer Engineering Computer Science University of Wisconsin, 2006.

[23] P. E. Agu, "Computer Graphics (CS/ECE 545) Lecture 7: Morphology (Part 2) \& Regions in Binary Images (Part 1)," Computer Science Dept. Worcester Polytechnic Institute (WPI). [Online]. Available: https://web.cs.wpi.edu/ emmanuel/courses/cs545/S14/slides/lecture07.pdf

[24] B. Girod, "Morphological Image Processing," Digital Image Processing, Stanford University, 2013.

[25] A. M. Namboodiri, "Morphological Image Processing," DIP: Monsoon, 2003. [Online]. Available: https://web.iiit.ac.in/ anoop/dip/DIP21Full.pdf

[26] S. Ravi and A. M. Khan, "Morphological operations for image processing: understanding and its applications," in Proc. 2nd National Conference on VLSI, Signal processing \& Communications NCVSComs-2013, 2013, pp. 1-6.

[27] E. R. Dougherty and R. A. Lotufo, "Hands-on Morphological Image Processing. Washington," USA: SPIE Publications, 2003, doi: 10.1117/3.501104.

[28] P. Srisombut, "Morphological Image Processing," in Graduate School of Information Sciences and Engineering, Tokyo Institute of Technology for IP seminar, vol. 4, 2004.

[29] A. Srikrishna, M. Pompapathi, and G. S. Rao, "Parametric based morphological transformation for contrast enhancement of color images in poor-lighting," Sadhana, vol. 40, no. 2, pp. 395-410, 2015, doi: 10.1007/s12046-015-0347-9.

[30] M. N. Farhan, M. G. Ayoub, H. M. Qassim, and A. K. Eesee, "Qualitative assessment of image enhancement algorithms for mammograms based on minimum EDV," TELKOMNIKA (Telecommunication, Computing, Electronics and Control), vol. 18, no. 2, pp. 928-935, 2020, doi: 10.12928/telkomnika.v18i2.14085. 


\section{BIOGRAPHIES OF AUTHORS}
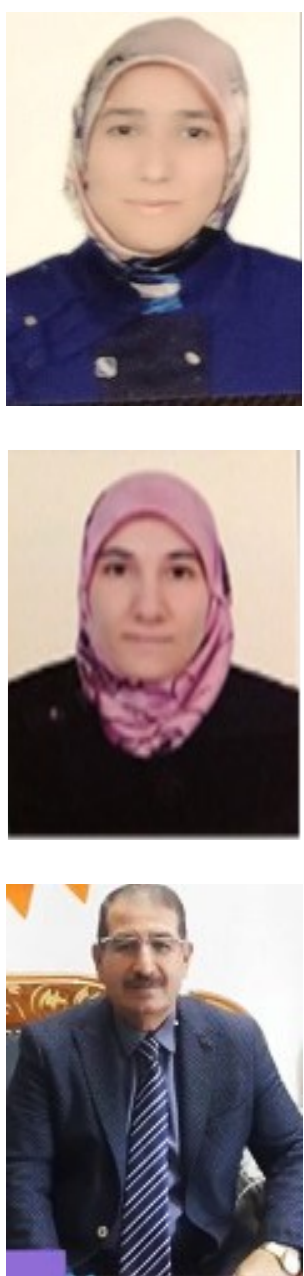

Hadeel Amjed Saeed is a member of the College of Computer Science and Information Technology as Assist. Teacher at University of Anbar, Anbar, Iraq. Received the B.Sc. (good) (second class) degree in computer science from the University of Baghdad, in 2007, and the M.Sc. degree in computer science from the University of Anbar, in 2011. She has published 3 refereed journal and conference papers. Her current research interests include mobile computing, artificial intelligence, Ad Hoc networks, security, and image processing and information technology.

Sumaya Hamad received the B.Sc. (good) (first class) degree in computer science from University of Anbar, Anbar, Iraq, in 2002, and the M.Sc. degree in computer science from University of Anbar, Anbar, Iraq, in 2012. She is currently PhD student in Computer Science Department at University of Technology, Baghdad, Iraq, and she an Assist. teacher. at the College of Computer Science and Information Technology, University of Anbar. She has published 7 refereed journal and conference papers. Her current research interests include mobile computing, artificial intelligence, Ad Hoc networks, search engines, and information technology.

Azmi Tawfik Hussain was born in Anbar-1961. Prof. Dr. in department of computer science in Collage of Computer Science and Information Technology/University of Anbar. He got on B.Sc of physical in Salhdee university 1983 - Iraq and M.Sc. in theoretical physics in al Yarmook university 1992-Jordan and Ph.D. in Image Processing from in Al-Mustinsary University-Iraq, (2001). He interesting in the follow filed (fractal geometry, image recognition, theoretical physics, signal processing). He supervised many PH.D. and Ms. Students in computer science and physics. He publishes more 25 papers in different conferences and scientific and scientific journals. 